\title{
Gestión del conocimiento y competencias profesionales de los docentes de una red educativa de Lima
}

\author{
Knowledge management and professional skills of the teachers from an \\ educative network of Lima \\ Manuel Torres V. ${ }^{1}$, Manuel Torres L., Marcelino Riveros Q., Rolando Solís N., Inés Acevedo H. \\ Universidad Nacional Mayor de San Marcos, Lima - Perú
}

RECIBIDO 08/06/2016, AcEPTADO 29/08/2016

\begin{abstract}
RESUMEN
En la medida en que la gestión del conocimiento ha adquirido una importancia significativa como factor de cambio y desarrollo en todo el quehacer de la sociedad, es clave crear una cultura donde la información y el conocimiento se valoren, se compartan, se gestionen y se usen eficaz y eficientemente. Bajo estas consideraciones, se desarrolla el presente trabajo de investigación que tiene como objetivo principal establecer si la gestión del conocimiento influye sobre las competencias profesionales en una muestra de docentes de una Red Educativa de Lima Metropolitana. Se utiliza un diseño correlacional causal, con una muestra de 217 docentes a quienes se les aplicó dos instrumentos de evaluación: el Cuestionario de Gestión del Conocimiento de Liliana Pedraja y el Inventario de competencias docentes de Úrsula Romaní. Los análisis a los que se sometieron estos instrumentos determinaron consistentes niveles de validez y confiabilidad.

Los resultados indican que la gestión del conocimiento influye significativamente sobre las competencias profesionales de los docentes $(\mathrm{F}=18,136, \mathrm{p}<, 001)$. Igualmente se puede apreciar en los resultados que las citadas variables están correlacionadas en sus totales y en sus dimensiones, por lo que podemos afirmar que se cumplieron todos los objetivos planteados.
\end{abstract}

Palabras clave: Gestión del conocimiento, competencias profesionales, desempeño laboral, compromiso institucional.

\begin{abstract}
As the knowledge management has acquired a significant importance as a change and development factor in all the work of the society, it is vital to create a culture where information and knowledge are valued, shared, managed and used effective and efficiently. Under these considerations, the current investigation is made, having as main objective to establish whether the knowledge management has influence on the professional skills in a sample of teachers from an Educative Network of Lima Metropolitana. A correlational causal design was used with a sample of 217 to whom two instruments of evaluation were
\end{abstract}

1 Profesor Principal de la Facultad de Psicología UNMSM. E-mail: manueltorres67@yahoo.es 
applied: the Questionnaire of Knowledge Management of Liliana Pedraja and the Inventory of Teaching skills of Úrsula Romaní. The analyses the instruments were submitted to determined consistent levels of validity and reliability.

The results show that the Knowledge Management has significant influence on the Professional skills of the teachers $(\mathrm{F}=18,136, \mathrm{p}<, 001)$. Moreover, it is possible to appreciate in the results that the cited variables are correlated in its totals and its dimensions, so it is possible to affirm that all the planted objectives were met.

Keywords: Knowledge management, professional skills, labour performance, institutional commitment.

\section{INTRODUCCIÓN}

Vivimos una nueva era en el desarrollo de la sociedad: la era de la información y el conocimiento, que ha cambiado nuestras formas de vida, tanto en lo personal como en lo organizacional. En este contexto, el activo intangible llamado conocimiento, se ha convertido en un tema de gran interés en tanto es vital para garantizar ventajas competitivas y la supervivencia de la organización, por lo que es un recurso que debe ser gestionado adecuadamente. En esta tarea se encuentran las diversas organizaciones sin importar el rubro a la cual pertenezcan.

En el plano educativo gestionar el conocimiento es parte de su quehacer diario; desde este punto de vista, Carneiro (2009) señala que en este terreno necesariamente se debe desarrollar y gestionar el conocimiento en tanto los profesores son por definición trabajadores del conocimiento: generan conocimiento, desarrollan y transfieren conocimiento a sus alumnos principalmente, quienes son personas en formación y dedicadas básicamente a aprender y a procesar conocimiento. Sin embargo, en la sociedad del conocimiento, las instituciones educativas no son las únicas gestoras de conocimiento, por lo que no tienen el monopolio del saber. Como señala Minakato Arceo (2009) existen otras entidades en las cuales se desarrollan competencias y adquieren conocimientos de manera espontánea y, con gran efectividad, se aprende con otros y de otros, se aprende en grupos de gran diversidad en edad, sexo, experiencia y cultura, en contextos reales y virtuales, en comunidades de práctica, etc.

Pero para las instituciones educativas la responsabilidad es mayor en tanto son organizaciones dedicadas al conocimiento, por lo que necesariamente se deben transformar en entes eficaces de gestión del conocimiento, pues de lo contrario no estarían cumpliendo el rol que la sociedad les ha asignado. En este proceso, las instituciones educativas deben utilizar todos los recursos posibles, particularmente las tecnologías de la información y la comunicación que se han convertido en instrumentos indispensables para el aprendizaje. Se debe reconocer que, en forma creciente, la creación de conocimiento involucra una alianza entre el conocimiento humano y la tecnología, de tal manera que las tecnologías de la información y comunicación permiten que el conocimiento directo generado por el hombre produzca conocimiento mejorado y extendido. 
Desde este punto de vista, queda claro que el conocimiento es el tesoro más preciado que pueda tenerse y buscarse, por lo que resulta necesario hacer circular este conocimiento con altos niveles de eficiencia. Esto implica acelerar los flujos de información desde los individuos hacia la organización para volver a los individuos y de esta manera producir un valor agregado para la organización en la producción del conocimiento. Siguiendo a Minakato Arceo (2009), la información se convierte, a través de los individuos, en un activo de conocimiento para la organización y este, a su vez, en un "activo de capital humano".

Lo señalado anteriormente deja claro la importancia que tiene el conocimiento y su adecuada gestión, por lo que la educación debe hacer todos los esfuerzos posibles a fin de poder generar, desarrollar, circular y utilizar el conocimiento. En estas circunstancias, aprender a aprender es fundamental en la sociedad del conocimiento y para ello el papel del docente es de importancia capital en la medida en que es el encargado de vincular al alumno con el conocimiento. En este proceso, las competencias profesionales del docente deben ser expresadas con total claridad, pues deben aplicar correctamente la metodología de enseñanza, fomentando la motivación propia del estudiante e incentivando su curiosidad. Esto significa que no es suficiente el dominio de la asignatura a enseñar, sino que también es necesario conocer y dominar aquellas actividades o técnicas pedagógicas que permitan transmitir los conocimientos de forma eficaz.

Como consecuencia de lo expuesto, los problemas de la presente investigación se plantean de la siguiente manera:

1. ¿Cuál es la influencia que tiene la Gestión del Conocimiento en las Competencias profesionales de los Docentes de una Red Educativa de Lima Metropolitana?

2. ¿Qué relación existe entre las dimensiones de la Gestión del Conocimiento y las Competencias profesionales de los Docentes de una Red Educativa de Lima Metropolitana?

3. ¿Qué relación existe entre las diversas dimensiones de la Gestión del Conocimiento y las diversas Competencias profesionales de los Docentes de una Red Educativa de Lima Metropolitana?

\section{OBJETIVOS}

1. Determinar la influencia que tiene la Gestión del Conocimiento en las Competencias profesionales de los Docentes de una Red Educativa de Lima Metropolitana.

2. Establecer la relación que existe entre las dimensiones de la Gestión del Conocimiento y las Competencias profesionales de los Docentes de una Red Educativa de Lima Metropolitana.

3. Establecer las relaciones que existen entre las diversas dimensiones de la Gestión del Conocimiento y las diversas Competencias profesionales de los Docentes de una Red Educativa de Lima Metropolitana. 


\section{HIPÓTESIS}

1. La Gestión del Conocimiento influye significativamente en las Competencias profesionales de los Docentes de una Red Educativa de Lima Metropolitana.

2. Existe una relación significativa entre las dimensiones de la Gestión del Conocimiento y las Competencias profesionales de los Docentes de una Red Educativa de Lima Metropolitana.

3. Existe una relación significativa entre las diversas dimensiones de la Gestión del Conocimiento y las diversas Competencias profesionales de los docentes de una Red Educativa de Lima Metropolitana.

\section{MÉTODOS Y RESULTADOS}

\section{Método}

De acuerdo con lo señalado por Sánchez y Reyes (2006), el método que corresponde en la presente investigación es el método descriptivo. Este método se desarrolla describiendo las situaciones y eventos, es decir cómo se manifiesta el fenómeno a investigar, ya que este busca especificar las propiedades importantes del problema en cuestión. El método descriptivo mide independientemente los conceptos y también pueden ofrecer la posibilidad de predicciones utilizando los procedimientos estadísticos correspondientes. A este tipo de investigaciones se les conoce como ex post facto, en tanto las variables independientes ya han ocurrido, por lo que no pueden ser manipuladas por el investigador.

\section{Diseño de investigación}

Para la selección del diseño de investigación se ha utilizado como base el libro de Hernández, Fernández y Baptista (2014). Según estos autores, el diseño adecuado para esta investigación es de tipo correlacional causal: "Los diseños correlacionales pueden limitarse a establecer relaciones entre variables sin precisar sentido de causalidad o pueden pretender analizar relaciones de causalidad. Cuando se limitan a relaciones no causales, se fundamentan en hipótesis correlacionales y cuando buscan evaluar relaciones causales, se basan en hipótesis causales".

\section{Muestra}

De acuerdo con Hernández, Fernández y Baptista (2014), el diseño de la muestra es probabilístico de tipo estratificado, clasificando a los docentes según las instituciones educativas de una Red de Aprendizaje de Lima Metropolitana. De la población objeto de estudio se seleccionó una muestra representativa mediante la técnica de muestreo aleatorio y por afijación proporcional. Para la estimación del tamaño de la muestra se utilizó la fórmula de Sierra Bravo (1994), la que dio como resultado un total de 217 docentes distribuidos de la siguiente forma: 
Tabla 1. Distribución de la muestra por Nivel Educativo

\begin{tabular}{ccc}
\hline Nivel & Frecuencia & Porcentaje \\
\hline Primaria & 106 & 48,8 \\
Secundaria & 111 & 51,2 \\
Total & 217 & 100,0 \\
\hline
\end{tabular}

\section{Instrumentos}

En la presente investigación se utilizaron dos instrumentos de recolección de datos:

$\checkmark$ Gestión del Conocimiento: Se utilizó el cuestionario de gestión del conocimiento de Liliana Pedraja.

$\checkmark$ Competencias profesionales docentes: Inventario de Competencias profesionales docentes de Úrsula Romaní.

\section{Análisis de validez y confiabilidad de los instrumentos}

En la medida en que se necesita garantizar la idoneidad de los instrumentos de evaluación, se procedió a realizar los análisis estadísticos respectivos que nos indiquen sus niveles de validez y confiabilidad. El análisis psicométrico del Cuestionario de gestión del conocimiento indica que la prueba alcanza un alfa de Cronbach de 0,97, lo que demuestra que el instrumento es confiable. El análisis psicométrico del Inventario de Competencias profesionales docente indica que la prueba alcanza un alfa de Cronbach de 0,93, lo que demuestra que el instrumento es confiable.

Los análisis de la validez de los instrumentos, realizados a través del Análisis Factorial Exploratorio, nos muestran que ambas pruebas presentan validez de constructo.

\section{RESULTADOS}

Los resultados del análisis exploratorio de los datos (ver tabla 2), en lo que se refiere a la forma de distribución, efectuado a través del test de bondad de ajuste a la curva normal de Kolmogorov-Smirnov, indica que, en todas las variables estudiadas, se obtienen coeficientes K-S Z que son estadísticamente significativos, por lo que se puede concluir que no se presentan adecuadas aproximaciones a la curva normal, por lo que es factible utilizar para el análisis de los datos, estadísticas no paramétricas. 
Tabla 2. Test de Bondad de Ajuste a la Curva Normal de Kolmogorov - Smirnov de las Variables estudiadas

\begin{tabular}{lllll}
\hline \multicolumn{1}{c}{ Variable } & Media & D. E. & K-SZ & Sig. \\
\hline Creación de conocimiento & 21,52 & 2,95 &, 162 &, 000 \\
Compartir conocimiento & 21,46 & 2,92 &, 172 &, 000 \\
Aplicar conocimiento & 21,34 & 2,78 &, 157 &, 000 \\
Creatividad en la organización & 21,73 & 2,83 &, 165 &, 000 \\
Innovación en la organización & 21,39 & 3,03 &, 171 &, 000 \\
Confianza en la organización & 21,46 & 2,86 &, 181 &, 000 \\
Información Interna & 21,33 & 2,98 &, 149 &, 000 \\
Información Externa & 21,47 & 3,07 &, 160 &, 000 \\
& & & & \\
Preparación de la enseñanza & 22,48 & 1,95 &, 152 &, 000 \\
Recursos del aprendizaje & 22,65 & 1,88 &, 108 &, 000 \\
Clima del aula de clase & 23,04 & 1,88 &, 116 &, 000 \\
Enseñanza para el aprendizaje & 22,65 & 2,03 &, 129 &, 000 \\
Evaluación del aprendizaje & 22,57 & 2,20 &, 092 &, 000 \\
Compromiso institucional & 22,10 & 2,51 &, 087 &, 000 \\
Relaciones Interpersonales & 23,55 & 1,83 &, 145 &, 000 \\
Responsabilidad docente & 24,25 & 1,17 &, 187 &, 000 \\
Ética docente & 24,44 & 1,04 &, 214 &, 000 \\
\hline
\end{tabular}

$\mathrm{N}=217$

\section{Análisis correlacionales causales}

Los resultados presentados en la tabla 3 indican que la correlación múltiple asciende a 0,80 , la cual da una $\mathrm{R}^{2}$ igual a 0,63 y que al ser corregida llega a 0,62 , lo cual indica que las variables predictoras permiten explicar el $62 \%$ de la varianza de la variable predicha que en este caso corresponde a las Competencias profesionales docentes.

Tabla 3. Resumen del modelo de Regresión Múltiple de las competencias profesionales docentes

\begin{tabular}{cccc}
\hline Modelo & $\mathrm{R}$ & R cuadrado & R cuadrado corregida \\
\hline 1 &, $80^{\mathrm{a}}$ &, 63 &, 62 \\
\hline
\end{tabular}

a. Variables predictoras: (constante), información externa, creatividad en la organización, aplicar conocimiento, confianza en la organización, información interna, compartir conocimiento, creación de conocimiento, innovación en la organización.

Los resultados del análisis de varianza indica que se alcanza un estadístico $\mathrm{F}=$ $44,622, \mathrm{p}=.001$ el cual es significativo. Este resultado permite concluir que la ecuación de regresión es estadísticamente significativa y permite predecir de forma consistente la variable predicha o dependiente, en este caso las Competencias profesionales docentes. 
Tabla 4. Análisis de Varianza de la ecuación de regresión múltiple de las Competencias profesionales docentes

\begin{tabular}{clccccc}
\hline Modelo & & Suma de cuadrados & gl & Media cuadrática & F & Sig. \\
\hline \multirow{4}{*}{1} & Regresión & 96287,935 & 8 & 12035,992 & 44,622 &, $000^{\mathrm{b}}$ \\
& Residual & 56104,259 & 208 & 269,732 & & \\
& Total & 152392,194 & 216 & & & \\
\hline
\end{tabular}

a. Variables predictoras: (Constante), Información Externa, Creatividad en la Organización, Aplicar Conocimiento, Confianza en la Organización, Información Interna, Compartir Conocimiento, Creación de Conocimiento, Innovación en la Organización.

b. Variable dependiente: Competencias profesionales docentes

\section{Análisis correlacionales descriptivos}

Los resultados presentados en la Tabla 5 nos indican que todas las dimensiones de la Gestión del Conocimiento se encuentran relacionadas significativamente con las Competencias profesionales de los Docentes.

Tabla 5. Análisis de Correlación (rho de Spearman) entre las dimensiones de la Gestión del Conocimiento y las Competencias profesionales de los Docentes

\begin{tabular}{lc}
\hline \multicolumn{1}{c}{ Variables } & Competencias profesionales \\
\hline Creación de conocimiento & $0,80^{* * *}$ \\
Compartir conocimiento & $0,74^{* * *}$ \\
Aplicar conocimiento & $0,76^{* * *}$ \\
Creatividad en la organización & $0,79^{* * *}$ \\
Innovación en la organización & $0,78^{* * *}$ \\
Confianza en la organización & $0,76^{* * *}$ \\
Información Interna & $0,78^{* * *}$ \\
Información Externa & $0,81 * * *$ \\
\hline
\end{tabular}

$* \mathrm{p}<.05 * * \mathrm{p}<.01 * * * \mathrm{p}<.001$

$\mathrm{N}=217$

El análisis correlacional efectuado con el estadístico rho de Spearman y presentados en la Tabla 6 nos indican que todas las dimensiones de ambas variables están correlacionadas entre sí de manera significativa. 
Tabla 6. Análisis de las Correlaciones entre las dimensiones de la Gestión del Conocimiento y las dimensiones de las Competencias Profesionales Docentes

\begin{tabular}{lccccccccc}
\hline \multicolumn{1}{c}{ Variables } & PEnse & Rapren & Clima & Eapren & Eval & ComIn & RRII & Rdoc & Etdoc \\
\hline $\begin{array}{l}\text { Creación de } \\
\text { conocimiento }\end{array}$ & $0,75^{* *}$ & $0,75^{* *}$ & $0,78^{* *}$ & $0,70^{* *}$ & $0,72^{* *}$ & $0,70^{* *}$ & $0,75^{* *}$ & $0,74^{* *}$ & $0,77^{* *}$ \\
$\begin{array}{l}\text { Compartir } \\
\text { conocimiento }\end{array}$ & $0,68^{* *}$ & $0,70^{* *}$ & $0,73^{* *}$ & $0,68^{* *}$ & $0,69^{* *}$ & $0,66^{* *}$ & $0,72^{* *}$ & $0,68^{* *}$ & $0,68^{* *}$ \\
$\begin{array}{l}\text { Aplicar } \\
\text { conocimiento }\end{array}$ & $0,69^{* *}$ & $0,72^{* *}$ & $0,73^{* *}$ & $0,72^{* *}$ & $0,71^{* *}$ & $0,65^{* *}$ & $0,73^{* *}$ & $0,69^{* *}$ & $0,71^{* *}$ \\
$\begin{array}{l}\text { Creatividad } \\
\text { organización }\end{array}$ & $0,74^{* *}$ & $0,76^{* *}$ & $0,76^{* *}$ & $0,72^{* *}$ & $0,72^{* *}$ & $0,69^{* *}$ & $0,74^{* *}$ & $0,74^{* *}$ & $0,74^{* *}$ \\
$\begin{array}{l}\text { Innovación } \\
\text { organización }\end{array}$ & $0,72^{* *}$ & $0,73^{* *}$ & $0,76^{* *}$ & $0,71^{* *}$ & $0,71^{* *}$ & $0,69^{* *}$ & $0,75^{* *}$ & $0,71^{* *}$ & $0,74^{* *}$ \\
$\begin{array}{l}\text { Confianza } \\
\text { organización }\end{array}$ & $0,72^{* *}$ & $0,72^{* *}$ & $0,73^{* *}$ & $0,70^{* *}$ & $0,69^{* *}$ & $0,68^{* *}$ & $0,73^{* *}$ & $0,67^{* *}$ & $0,69^{* *}$ \\
$\begin{array}{l}\text { Información } \\
\text { Interna }\end{array}$ & $0,70^{* *}$ & $0,72^{* *}$ & $0,76^{* *}$ & $0,72^{* *}$ & $0,73^{* *}$ & $0,68^{* *}$ & $0,78^{* *}$ & $0,73^{* *}$ & $0,73^{* *}$ \\
$\begin{array}{l}\text { Información } \\
\text { Externa }\end{array}$ & $0,75^{* *}$ & $0,75^{* *}$ & $0,80^{* *}$ & $0,75^{* *}$ & $0,75^{* *}$ & $0,71^{* *}$ & $0,79^{* *}$ & $0,75^{* *}$ & $0,76^{* *}$ \\
\hline
\end{tabular}

$* \mathrm{p}<, 05 * * \mathrm{p}<, 01 * * * \mathrm{p}<, 001$

$\mathrm{N}=217$

\section{DISCUSIÓN}

En lo que respecta a la primera hipótesis planteada, "La Gestión del Conocimiento influye significativamente en las Competencias profesionales de los Docentes de una Red Educativa de Lima Metropolitana", los resultados nos muestran que existen relaciones causales entre estas variables, por lo que se debe darles toda la importancia que el caso requiere en la medida en que pueden contribuir significativamente a lograr instituciones educativas realmente de calidad. Así mismo, se muestra la existencia de correlaciones entre las diversas dimensiones de ambas variables, lo que nos indica que dichas variables están bastante unidas, es decir que marchan en la misma dirección, por lo que hay necesidad de prestarles la debida atención al momento de tomar medidas tendientes al desarrollo de las instituciones educativas.

Se debe señalar, también, que estos resultados se encuentran en la misma dirección que los reportados por investigadores como Pedraglio (2013), Pullch (2005), Fuentes-Morales (2009), Morales Morgado (2008) y Godoy (2006), quienes resaltan la importancia que tiene hoy en día la gestión del conocimiento y las competencias profesionales en las diversas instituciones, particularmente las educativas. En esta línea, Cornella (2000) y Paños (1999) señalan que la gestión del conocimiento es un proceso que debe iniciarse en los centros educativos para ayudar a identificar, seleccionar, organizar, diseminar y transferir información y 
experiencia. En este proceso, la docencia debe aprender a identificar y manejar el conocimiento de tal manera que pueda utilizarlo en provecho de la institución y de los propios estudiantes.

En lo que se refiere a las hipótesis específicas, tenemos que todas las dimensiones de la gestión del conocimiento se encuentran relacionadas a las competencias profesionales de los docentes, resaltando de manera importante lo siguiente:

En lo que se refiere a la creación de conocimiento, los teóricos coinciden en que es una de las competencias que más se ha trabajado en tanto son las que preparan para el aprendizaje a lo largo de la vida (O'Sullivan, 2002). Desde este punto de vista, formar a los docentes para interactuar de forma efectiva con la información es un reto imprescindible en el marco de la implementación de programas de gestión del conocimiento. Así, en diversos estudios que identifican las habilidades y capacidades de los docentes de la era del conocimiento, la creación de conocimiento se ha considerado una competencia básica para la competitividad de las instituciones educativas (Abell, 1999).

Por otra parte, en el caso de compartir conocimiento, es importante resaltar lo que Nonaka y Takeuchi (2001) señalan en el sentido de que para que se efectúe la transmisión del conocimiento los académicos deben hacer llegar el conocimiento explícito a los profesionales quienes lo llevarán a la práctica para posteriormente retroalimentar a los académicos con los resultados, valiéndose de un lenguaje común y a través de los medios adecuados.

Respecto de la innovación organizacional, se asume que la supervivencia de las organizaciones depende en gran medida de su posibilidad de cambio, su capacidad de adaptación a las demandas del entorno y de su flexibilidad para afrontar la incertidumbre, lo cual a su vez, depende de la forma en que las organizaciones son lideradas. Schermerhorn, Hunt, y Osborn (2004) señalan que la evidencia ha demostrado que la velocidad de los cambios en la actualidad es cada vez mayor, lo cual implica que las posibles soluciones que se plantean para enfrentarlos tendrán una menor duración, fenómeno que el autor ha llamado el problema del ciclo de vida de las soluciones. En ocasiones, los cambios frecuentes se relacionan con logros a corto plazo; sin embargo no se evalúa el efecto que estos tienen a largo plazo, principalmente sobre la percepción de las personas, y su capacidad de adaptación. Los líderes deben encontrar el adecuado balance entre estas ganancias y sus efectos.

Por otra parte, en lo que respecta a la información interna, esta debe ser considerada como una herramienta estratégica clave en las instituciones y es que para aumentar la eficacia del equipo humano, verdadero artífice de los resultados, ha de sentirse a gusto e integrado dentro de su organización y esto solo es posible si los trabajadores están informados, conocen los diferentes entramados de la compañía, su misión, su filosofía, sus valores, su estrategia, se sienten parte de ella y, por consiguiente, están dispuestos a dar todo de sí mismos (Escobar, 1997), por lo que su adecuado manejo al interior de las instituciones educativas debe permitir mejorar la actividad profesional de los docentes. 
Finalmente, en el caso de la información externa, se debe tener claro que la información produce rendimientos, ya que tiene la misión de informar, revelar alternativas, reducir incertidumbres y procurar soluciones entre otras cosas (Hornos, Araque y Abad, 1998). Este rendimiento es más importante o relevante de lo que parece, ya que ayuda a la toma de decisiones y es que cada día las empresas enfrentan nuevos desafíos: deben alcanzar niveles máximos de calidad y satisfacción de sus clientes, lanzar nuevos productos, posicionar y consolidar los ya existentes, buscar mayor participación de mercado, etc.

\section{CONCLUSIONES}

1. Los resultados alcanzados nos indican que la Gestión del conocimiento influye significativamente en las Competencias profesionales de los Docentes de una Red Educativa de Lima Metropolitana.

2. Los resultados correlacionales alcanzados nos indican que existen relaciones significativas entre las dimensiones de la gestión del conocimiento y las Competencias profesionales de los Docentes.

3. Los resultados correlacionales alcanzados nos indican que existen relaciones significativas entre las diversas dimensiones de la Gestión del Conocimiento y las diversas Competencias profesionales de los docentes.

\section{REFERENCIAS BIBLIOGRÁFICAS}

Abell, A. (1999). Skills for the Knowledge Management: a briefing paper by TFLP. Londres: TFLP Ltd.

Carneiro, R., Toscano, J.C., Díaz, T. (2009). Los desafíos de las TIC para el cambio educativo. Madrid: OEI. Fundación Santillana.

Coll, C. (2007). Las competencias en la educación escolar: algo más que una moda y mucho menos que un remedio. Rev. Innovación Educativa. Departamento de Psicología Evolutiva y de la Educación. Facultad de Psicología. Universidad de Barcelona

Cornellá, A. (2000). Infonomia.com. La empresa es información. Bilbao: Deusto.

Escobar, B. (1997). La evaluación económica de los sistemas de información. Sevilla: Edit. Universidad de Sevilla.

Fuentes-Morales, B. (2009). La gestión de conocimiento en las relaciones académico-empresariales. Un nuevo enfoque para analizar el impacto del conocimiento académico. Tesis Doctoral. Universidad Politécnica de Valencia.

Godoy, M. (2006). Constructos teóricos que fundamentan las competencias del docente universitario para la gestión del conocimiento en contextos virtuales de aprendizaje. Tesis Doctoral. Universidad de los Andes. 
Hernández, Fernández y Baptista (2014). Metodología de la Investigación. México: Ed McGraw-Hill.

Hornos, M., Araque, F. y Abad, M. (1998). La gestión de la información como clave para adquirir ventaja competitiva: los MIS. En Alta Dirección. Número 199. Mayo. Barcelona.

Minakato Arceo, A. (2009). Gestión del conocimiento en educación y transformación de la escuela. Notas para un campo en construcción. México. Sinéctica, revista electrónica de educación. http://www.oei.es/mx43.htm

Morales Morgado, E.M. (2008). Gestión del Conocimiento en Sistemas e-learning, Basado en Objetos de Aprendizaje Cualitativa y Pedagógicamente definidos. Colección Vítor 273. Ediciones Universidad de Salamanca y Erla Mariela Morales Morgado.

Nonaka, I. y Takeuchi, H. (1995). La organización creadora de conocimiento. México: Oxford University Press, .

O'Sullivan, C. (2002). Is information literacy relevant in the real world? Reference Services Review. Vol. 30, núm. 1, págs. 7-14.

Paños, A. (1999). Reflexiones sobre el papel de la información como recurso competitivo de la empresa. Anales de Documentación, no 2, pp. 21-38

Pedraglio, D. (2013). Gestión del conocimiento en el sector construcción. Tesis Magíster. Universidad Peruana de Ciencias Aplicadas.

Perrenoud, P. (2004). Diez nuevas competencias para enseñar. Barcelona: Graó.

Pullch, J. (2005). Modelo de gestión del conocimiento para empresas constructoras. Tesis de Magíster. Universidad Nacional de Ingeniería.

Sánchez, H. y Reyes, C. (2006). Metodología y diseño de la investigación científica. Lima: Editorial Visión Universitaria.

Schermerhorn, J., Hunt, J., y Osborn, R. (2004). Comportamiento organizacional. México: Editorial Limusa. 